\title{
Quantum probes to experimentally assess correlations in a composite system
}

\author{
Andrea Smirne* \\ Dipartimento di Fisica, Università degli Studi di Milano, I-20133 Milano, Italy, \\ Dipartimento di Fisica, Università degli Studi di Trieste, I-34127 Trieste, Italy, and INFN, Sezione di Milano, I-20133 Milano, Italy \\ Simone Cialdi ${ }^{\dagger}$ \\ Dipartimento di Fisica, Università degli Studi di Milano, I-20133 Milano, Italy and INFN, Sezione di Milano, I-20133 Milano, Italy \\ Giorgio Anelli \\ Dipartimento di Fisica, Università degli Studi di Milano, I-20133 Milano, Italy \\ Matteo G. A. Paris ${ }^{\ddagger}$ \\ Dipartimento di Fisica, Università degli Studi di Milano, I-20133 Milano, Italy and CNISM, Udr Milano, I-20133 Milano, Italy \\ Bassano Vacchini ${ }^{\S}$ \\ Dipartimento di Fisica, Università degli Studi di Milano, I-20133 Milano, Italy and INFN, Sezione di Milano, I-20133 Milano, Italy \\ (Received 28 January 2013; published 11 July 2013)
}

\begin{abstract}
We suggest and demonstrate a technique to obtain relevant information about a composite system by performing measurements on only a small and easily accessible part of it, which we call a quantum probe. We show in particular how quantitative information about the angular correlations of pairs of entangled photons generated by spontaneous parametric down-conversion may be accessed through the study of the trace distance between two polarization states evolved from different initial conditions. After estimating the optimal polarization states to be used as the quantum probe, we provide a detailed analysis of the connections between the increase of the trace distance above its initial value and the amount of angular correlation.
\end{abstract}

DOI: 10.1103/PhysRevA.88.012108

PACS number(s): 03.65.Yz, 03.65.Ta, 42.50.Dv

\section{INTRODUCTION}

The control of quantum systems plays a basic role in the experimental investigation of the predictions of quantum theory as well as in the development of quantum technologies for applications. Indeed, great attention has been recently paid to engineering the dynamics of quantum systems in order to properly generate, manipulate, and exploit significant quantum features [1-8].

Consider a large quantum system whose full characterization is only partially feasible or requires a measurement scheme which is experimentally challenging. In such a case, it is crucial to develop effective strategies in order to assess relevant pieces of information about the overall system by monitoring only a small part, which then acts as a probe. A natural procedure is to control the interaction of the small subsystem with the rest of the total system in such a way that the former can encode the information of interest. Here, we provide an explicit example of this strategy in an all-optical setup, where the system under study consists of entangled pairs of photons generated by spontaneous parametric down-conversion (SPDC) [9-12]. By properly engineering the interaction between the polarization and momentum degrees of freedom of the photons via a one-dimensional spatial light modulator (SLM), we can access some information regarding the momentum correlations

\footnotetext{
*andrea.smirne@unimi.it

${ }^{\dagger}$ simone.cialdi@mi.infn.it

${ }^{\ddagger}$ matteo.paris@ fisica.unimi.it

§assano.vacchini@mi.infn.it
}

between the two photons by simply performing visibility measurements on the polarization degrees of freedom.

As specific figure of merit, we exploit the trace distance between polarization states. As we shall see, an increase of the trace distance above its initial value reflects the transfer of information from the momentum (i.e., angular) degrees of freedom to the polarization ones due to the engineered interaction. The trace-distance analysis of quantum dynamics has been recently introduced, leading to important results concerning the non-Markovianity of a quantum dynamics [13-18], the characterization of the presence of initial correlations between the quantum system and its environment [19-22], the relevance of nonlocal memory effects [23-25], and reservoir engineering in ultracold gases [26-28].

The paper is structured as follows. In the next section we describe the physical system we are going to investigate and present the details of the experimental apparatus. In Sec. III we illustrate the trace-distance approach to the dynamics of an open system and present the details of the calculations of its evolution for different angular and polarization states. Section IV is devoted to illustrating the experimental results about the optmization of the probe and the link between the behavior of the trace distance and the initial correlations in the angular degrees of freedom. Finally, Sec. V closes the paper with some concluding remarks.

\section{THE PHYSICAL SYSTEM AND THE EXPERIMENTAL APPARATUS}

Our overall system consists of pairs of entangled photons generated by SPDC in a two-crystal geometry [11]. The pairs 
are detected along two beams, named the signal and idler, which are centered around the directions fixed by the phasematching condition. The two-photon state generated by SPDC can be written

$$
\begin{aligned}
|\psi\rangle= & \int d \omega_{p} d \omega_{s} d \theta_{s} d \theta_{i} A\left(\omega_{p}\right) \tilde{F}\left(\Delta k_{\perp}\right) \operatorname{sinc}\left(\Delta k_{\|} L / 2\right) \\
& \times\left[\cos \alpha\left|H, \theta_{s}, \omega_{s}\right\rangle\left|H, \theta_{i}, \omega_{p}-\omega_{s}\right\rangle\right. \\
& \left.+e^{i \Phi\left(\omega_{p}, \theta_{s}, \theta_{i}\right)} \sin \alpha\left|V, \theta_{s}, \omega_{s}\right\rangle\left|V, \theta_{i}, \omega_{p}-\omega_{s}\right\rangle\right]
\end{aligned}
$$

where up to first order in frequency and angle, we have

$$
\begin{aligned}
\Delta k_{\|} & =-\frac{\omega_{p}^{0} \theta^{0}}{2 c}\left(\theta_{s}+\theta_{i}\right), \\
\Delta k_{\perp} & =\frac{\omega_{p}^{0}}{2 c}\left(\theta_{s}-\theta_{i}\right)+\frac{2 \theta^{0} \omega_{s}}{c} .
\end{aligned}
$$

Here, $\omega_{p}$ is the shift of the pump frequency with respect to the central frequency $\omega_{p}^{0}(405 \mathrm{~nm}), \theta_{s}$ and $\omega_{s}\left(\theta_{i}\right.$ and $\omega_{i}=$ $\left.\omega_{p}-\omega_{s}\right)$ are the signal (idler) angle and frequency shift with respect to the phase-matching condition,

$$
\theta_{s}^{0}=\theta_{i}^{0} \equiv \theta^{0}=3^{\circ}, \quad \omega_{s}^{0}=\omega_{i}^{0}=\omega_{p}^{0} / 2,
$$

while $|P, \theta, \omega\rangle$ denotes the single-photon state with polarization $P=H, V$, angle $\theta$, and frequency $\omega$. Moreover, $A\left(\omega_{p}\right)$ is the spectral amplitude of the pump, $\tilde{F}\left(\Delta k_{\perp}\right)$ is the Fourier transform of its spatial amplitude, and the $\operatorname{sinc}\left(\Delta k_{\|} L / 2\right)$ function arises due to the finite crystal size $(L=1 \mathrm{~mm})$ along the longitudinal direction. The two-crystal geometry implies that the polarization degrees of freedom of the two photons are entangled and it further introduces the phase term $\Phi\left(\omega_{p}, \theta_{s}, \theta_{i}\right)$, which is due to the different optical paths followed by the pairs of photons generated in the first and in the second crystal [29-31]. Up to first order this term may be written as

$$
\Phi\left(\omega_{p}, \theta_{s}, \theta_{i}\right) \simeq \Phi_{0}+\Delta \tau \omega_{p}+\kappa \theta_{s}+\eta \theta_{i}
$$

Finally, the probabilities of generating $|V V\rangle$ or $|H H\rangle$ photons, $\sin ^{2} \alpha$ and $\cos ^{2} \alpha$, respectively, are determined by the polarization of the incident laser and may be tuned at will.

The overall state in Eq. (1) contains the full information about the angular correlations between the signal and the idler beams. By properly engineering the two-photon evolution, relevant information about these angular correlations gets encoded into the polarization degrees of freedom and then can be easily accessed. In fact, through the SLM we can impose an arbitrary polarization- and position-dependent phase shift on the two-photon state in Eq. (1). On the one hand, a linear phase

$$
\bar{\Phi} \equiv-\Phi_{0}-\kappa \theta_{s}-\eta \theta_{i}
$$

is set to compensate (offset) the corresponding terms in the first-order expansion of $\Phi\left(\omega_{p}, \theta_{s}, \theta_{i}\right)$ [29-32]. On the other hand, a further linear phase on both signal and idler beams may be imposed, to emulate a time evolution of the two-photon state [33] in the spatial domain.

The experimental setup is shown in Fig. 1. A linearly polarized $\mathrm{cw} 405 \mathrm{~nm}$ diode laser (Newport LQC405-40P) passes through two cylindrical lenses, which compensate beam astigmatism (AC), and then through a spatial filter (SF)

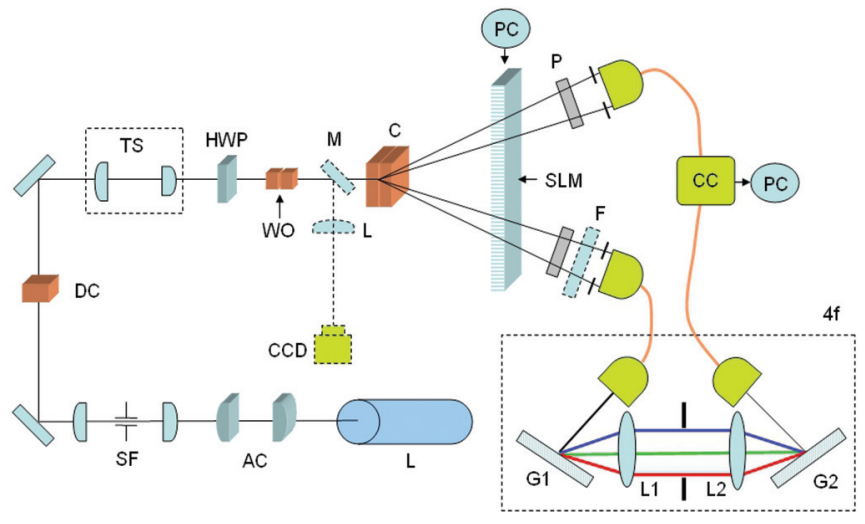

FIG. 1. (Color online) Schematic diagram of the experimental setup. A linearly polarized cw $405 \mathrm{~nm}$ diode laser passes through two cylindrical lenses to compensate beam astigmatism (AC), a spatial filter (SF) to obtain a Gaussian profile, and a telescopic system (TS) to adjusts the radius and the divergence of the beam. Two $\beta$-barium borate crystals (C, length $1 \mathrm{~mm}$ ), cut for type-I down-conversion and with optical axes aligned in perpendicular planes, are the source of polarization- and momentum-entangled photon pairs with $\theta^{0}=3^{\circ}$. A compensation crystal on the pump (DC) imposes a delay time between the vertical and horizontal polarization, and two thin crystals $(0.5 \mathrm{~mm})$ ensure spatial walk-off compensation (WO). An interference filter or a long-pass filter, (both denoted by F) may be put on the signal path to select the spectral width of the radiation (10 or $45 \mathrm{~nm}$ ). The spectral width and profile are tuned by a $4 f$ optical system after the coupler on the signal path, which consists of two gratings (G1 and G2) with 1200 lines $/ \mathrm{mm}$, and two achromatic lenses (L1 and L2) with $f=$ $35 \mathrm{~mm}$. Spatial modulation is imposed by a liquid crystal phase mask $(\mathrm{SLM})\left(64 \times 10 \mathrm{~mm}^{2}\right)$ divided into 640 horizontal pixels each $d=100 \mu \mathrm{m}$ wide and placed at $310 \mathrm{~mm}$ from the crystals. When the mirror (M) is switched into the radiation path, a cylindrical lens (L) generates the Fourier transform profile of the pump at its focal distance $(1 \mathrm{~m})$, where a CCD camera is located. Two polarizers $(\mathrm{P})$ is used to measure the visibility of the entangled state.

composed of two lenses and a pinhole in the Fourier plane to obtain a Gaussian profile by removing the multimode spatial structure of the laser pump. Finally, a telescopic system (TS) prepares a beam with the proper radius and divergence. Two $1 \mathrm{~mm} \beta$-barium borate crystals (C), cut for type-I downconversion, with optical axes aligned in perpendicular planes, are used as a source of polarization- and momentum-entangled photon pairs with $\theta^{0}=3^{\circ}$. We use a compensation crystal on the pump (DC) [34], which acts on the delay time between the vertical and horizontal polarization, and two thin crystals $(0.5 \mathrm{~mm})$ for the spatial walk-off compensation (WO).

An interference filter or a long-pass filter (both denoted by $\mathrm{F}$ in Fig. 1) may be inserted in the signal path to select the spectral width of the radiation ( 10 or $45 \mathrm{~nm}$ ). In order to obtain different spectral widths or a particular spectral profile, we use a $4 f$ optical system after the coupler on the signal path. The $4 f$ system consists of two gratings (G1 and G2) of 1200 lines $/ \mathrm{mm}$ and two achromatic lenses (L1 and L2) with $f=35 \mathrm{~mm}$. The distance between the lenses and the grating is $f$ and the distance between the two lenses is $2 f$. In this configuration, in between the two lenses the spectral components are focalized and well separated, so that it is possible to put a slit here to select the wanted spectral width. 
Spatial light modulation is achieved by a liquid-crystal phase mask (dimension $64 \times 10 \mathrm{~mm}^{2}$ ) divided into 640 horizontal pixels each $d=100 \mu \mathrm{m}$ wide. The SLM is placed before the detectors, at $310 \mathrm{~mm}$ from the generating crystals, to purify the state and to introduce the desired spatial phase function. When the mirror $(\mathrm{M})$ is switched into the radiation path a cylindrical lens (L) generates the Fourier transform profile of the pump at its focal distance $(1 \mathrm{~m})$, where a CCD camera is located. Two polarizers $(\mathrm{P})$ are used to measure the visibility of the entangled state.

\section{TRACE-DISTANCE ANALYSIS}

The trace distance between two quantum states $\rho^{1}$ and $\rho^{2}$ is defined as

$$
D\left(\rho^{1}, \rho^{2}\right)=\frac{1}{2} \operatorname{Tr}\left|\rho^{1}-\rho^{2}\right|=\frac{1}{2} \sum_{k}\left|x_{k}\right|,
$$

where the $x_{k}$ 's are the eigenvalues of the traceless operator $\rho^{1}-\rho^{2}$. The trace distance is a metric on the space of physical states and thus $0 \leqslant D\left(\rho^{1}, \rho^{2}\right) \leqslant 1$. The physical meaning of the trace distance lies in the fact that it measures the distinguishability between two quantum states [35]. As a consequence, given an open quantum system $S$ interacting with an environment $E$ [36], any variation of the trace distance of two open system's states $D\left(\rho_{s}^{1}(t), \rho_{s}^{2}(t)\right)$ can be read in terms of an exchange of information between the open system and the environment $[13,17,19]$. Here, $\rho_{s}^{1}(t)$ and $\rho_{s}^{2}(t)$ are the open system's states evolved from different initial total states $\rho_{S E}^{1}(0)$ and $\rho_{S E}^{2}(0)$ through the relation

$$
\rho_{S}^{k}(t)=\operatorname{tr}_{E}\left\{U(t) \rho_{S E}^{k}(0) U^{\dagger}(t)\right\}, \quad k=1,2,
$$

where the total system $S E$ is assumed to be closed and hence evolves through a unitary dynamics $U(t)$ [36]. In particular, if there are no initial system-environment correlations, i.e.,

$$
\rho_{S E}^{k}(0)=\rho_{S}^{k}(0) \otimes \rho_{E}^{k}(0), \quad k=1,2,
$$

then an increase of the trace distance above its initial value,

$$
D\left(\rho_{s}^{1}(t), \rho_{s}^{2}(t)\right)>D\left(\rho_{s}^{1}(0), \rho_{s}^{2}(0)\right),
$$

witnesses the difference of the two initial environmental states, i.e., $\rho_{E}^{1}(0) \neq \rho_{E}^{2}(0)[19,37]$. This relation already shows how the trace distance between an open system's states allows one to access nontrivial information regarding the environment. In the following we further elaborate on this point, and present a quantitative link between the trace-distance behavior and the amount of environmental correlation.

In view of the trace-distance analysis, our physical system can be characterized as follows. The polarization degrees of freedom are the open system $S$ and the angular degrees of freedom the corresponding environment $E$. The latter are in turn manipulated by varying the divergence of the pump, as well as by selecting the frequency-spectrum width of the two-photon state generated by SPDC. We therefore study the evolution of the trace distance between two polarization states evolved from different initial $S E$ states. These initial $S E$ states are product states since the compensating phase imposed by the SLM decouples the polarization degrees of freedom from the rest of the system. In particular, we investigate how the trace-distance evolution of the polarization

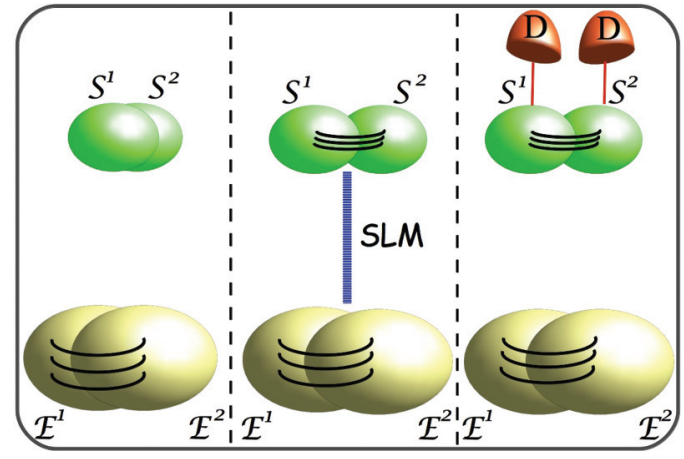

FIG. 2. (Color online) Logical scheme of the experiment. In the first stage the system and environment are uncorrelated, and the environmental states ( $E^{1}$ and $E^{2}$ ) differ due to internal correlations. The wiring represents the information about these different correlations. In the second stage the system and environment are coupled through the SLM, so that information on the environmental correlations is transferred to the system states $S^{1}$ and $S^{2}$, making them more distinguishable than the initial ones. Finally, the system is read out by the detector (D), acting on the system only, in the third and final stage. Note that the two states refer to two distinct runs of the experiment.

degrees of freedom, which are a small and easily accessible component of the total system, is sensitive to the different angular correlations within $\rho_{E}^{1}$ and $\rho_{E}^{2}$, thus allowing this characteristic feature of the overall two-photon state to be assessed. A logical scheme of the experiment is depicted in Fig. 2.

Let us emphasize that our apparatus exploits all the degrees of freedom of the photons generated by SPDC: the polarization degrees of freedom as the open system, the angles as the environment, and the frequencies, together with the spatial properties of the pump, as a tool to tune the correlations within the environment.

\section{A. Trace-distance evolution for different angular and polarization states}

In our apparatus, the angular state after the compensation of the phase through the SLM is described by the density matrix

$$
\rho_{E}=\int d \boldsymbol{\theta} d \boldsymbol{\theta}^{\prime} h\left(\boldsymbol{\theta} ; \boldsymbol{\theta}^{\prime}\right)|\boldsymbol{\theta}\rangle\left\langle\boldsymbol{\theta}^{\prime}\right|,
$$

where $\boldsymbol{\theta} \equiv\left(\theta_{s}, \theta_{i}\right)$ and

$$
h\left(\boldsymbol{\theta} ; \boldsymbol{\theta}^{\prime}\right) \equiv \operatorname{sinc}(\boldsymbol{\theta}) \operatorname{sinc}\left(\boldsymbol{\theta}^{\prime}\right) \int_{\Omega_{s}} d \omega_{s} \tilde{F}\left(\Delta k_{\perp}\right) \tilde{F}^{*}\left(\Delta k_{\perp}^{\prime}\right) .
$$

The influence of the pump spectrum on the angular state can be neglected [30,32] and the integration over $\omega_{s}$ is performed on the frequency interval $\Omega_{s}$ selected by the filter or by the $4 f$ setup on the signal path. The joint probability distribution $P(\boldsymbol{\theta}) \equiv h(\boldsymbol{\theta} ; \boldsymbol{\theta})$ determines the angular correlations, which can be quantified as

$$
C=\frac{\left\langle\theta_{s} \theta_{i}\right\rangle-\left\langle\theta_{s}\right\rangle\left\langle\theta_{i}\right\rangle}{\sqrt{V_{s} V_{i}}},
$$

with $V_{j}=\left\langle\theta_{j}^{2}\right\rangle-\left\langle\theta_{j}\right\rangle^{2}$ the variance of the angular distribution $P\left(\theta_{j}\right), j=s, i$. In particular, given a collimated beam with a large pump waist, so that the transverse momentum is nearly 
conserved, the signal and idler angles are the more correlated the narrower is the selected frequency spectrum. On a similar footing, for a $10 \mathrm{~nm}$ spectrum and a fixed pump waist, the correlation of the angular degrees of freedom grows with decreasing pump divergence. Thus, we can control the initial correlations of the environment by selecting the frequency spectrum of the two-photon state or the divergence of the pump. The polarization state after the purification and before the interaction with the environment reads

$$
\rho_{S}(0)=\gamma|\psi\rangle\langle\psi|+(1-\gamma) \rho^{m},
$$

where

$$
|\psi\rangle=\cos \alpha|H H\rangle+\sin \alpha|V V\rangle
$$

[see Eq. (1)] is a pure entangled state and

$$
\rho^{m}=\cos ^{2} \alpha|H H\rangle\left\langle H H\left|+\sin ^{2} \alpha\right| V V\right\rangle\langle V V|
$$

the corresponding mixture. In our apparatus, we can tune $\alpha$ by using the polarization of the pump, and $\gamma$ by changing the crystal throughout the pump, which precompensates the delay time due to the two-crystal geometry [34]. The initial purity $p=\operatorname{Tr} \rho_{s}^{2}(0)$ is given by

$$
p=1-\frac{1}{2}\left(1-\gamma^{2}\right) \sin ^{2}(2 \alpha),
$$

whereas its concurrence $\mathcal{C}$ [38] reads

$$
\mathcal{C}=\gamma|\sin (2 \alpha)| \text {. }
$$

In the following, we will compare the evolution of polarization states evolved from different initial states $\rho_{S}^{k}(0) \otimes \rho_{E}^{k}(0), k=$ 1,2 . The two initial open system states $\rho_{s}^{k}(0)$ have polarization parameters $\alpha_{k}$ and $\gamma_{k}$ [see Eqs. (8) and (9)] while the two initial environmental states $\rho_{E}^{k}(0)$ have angular amplitudes $h_{k}\left(\boldsymbol{\theta} ; \boldsymbol{\theta}^{\prime}\right)$ [see Eqs. (5) and (6)] and thus joint angular probability distributions $P_{k}(\boldsymbol{\theta})=h_{k}(\boldsymbol{\theta} ; \boldsymbol{\theta})$. The system-environment interaction is driven by the SLM. More specifically, the SLM imposes a linear phase function corresponding to the unitary operator

$$
U(\beta)\left|V, \theta_{s}\right\rangle\left|V, \theta_{i}\right\rangle=e^{i \beta\left(\theta_{s}-\theta_{i}\right)}\left|V, \theta_{s}\right\rangle\left|V, \theta_{i}\right\rangle,
$$

where $\beta$ is the evolution parameter. The evolved polarization states are thus given by

$$
\begin{aligned}
\rho_{S}^{k}(\beta) & =\operatorname{Tr}\left[U(\beta) \rho_{S}^{k}(0) \otimes \rho_{E}^{k}(0) U^{\dagger}(\beta)\right] \\
& =\frac{\epsilon_{k}(\beta)}{\sin \left(2 \alpha_{k}\right)}\left|\psi_{k}\right\rangle\left\langle\psi_{k}\right|+\left(1-\frac{\epsilon_{k}(\beta)}{\sin \left(2 \alpha_{k}\right)}\right) \rho_{k}^{m},
\end{aligned}
$$

where $\left|\psi_{k}\right\rangle=\cos \alpha_{k}|H H\rangle+\sin \alpha_{k}|V V\rangle$ and

$$
\epsilon_{k}(\beta)=\gamma_{k} \sin \left(2 \alpha_{k}\right) \int d \theta_{s} d \theta_{i} e^{i \beta\left(\theta_{s}-\theta_{i}\right)} P_{k}\left(\theta_{s}, \theta_{i}\right)
$$

is a real function of $\beta$, as follows from the fact that the joint probability distribution is symmetric under the exchange $\theta_{s} \leftrightarrow \theta_{i}$. It is worth emphasizing that the absolute value of $\epsilon_{k}(\beta)$ equals the concurrence as well as the interferometric visibility of the state $\rho_{s}^{k}(\beta)$. In particular, we measure the visibility by counting the coincidences with polarizers set at $45^{\circ}, 45^{\circ}$ and at $45^{\circ},-45^{\circ}$; see [39] for further details. Moreover, by virtue of the specific evolution obtained through the SLM, $\epsilon_{k}(\beta)$ is fixed by the Fourier transform of the spatial profile $\left|\tilde{F}\left(\Delta k_{\perp}\right)\right|^{2}$, which at first order is a function of $\theta_{s}-\theta_{i}$ and $\omega_{s}$ [see Eq. (2)], thus depending on both the pump divergence and the selected frequency spectrum. Thus the engineered evolution [see Eq. (12)] guarantees that the interferometric visibility is sensitive to the different angular correlations in the environment.

Finally, the trace distance $D(\beta) \equiv D\left(\rho_{S}^{1}(\beta), \rho_{S}^{2}(\beta)\right)$ between the polarization states is given by

$$
D(\beta)=\sqrt{\left(\cos ^{2} \alpha_{1}-\cos ^{2} \alpha_{2}\right)^{2}+\left[\epsilon_{1}(\beta)-\epsilon_{2}(\beta)\right]^{2} / 4}
$$

as follows from Eqs. (3) and (13).

\section{EXPERIMENTAL RESULTS}

\section{A. Characterization of the probe}

As a first step, we show how the choice of the initial polarization states $\rho_{s}^{1}(0)$ and $\rho_{s}^{2}(0)$ influences in a critical way whether the subsequent trace-distance evolution is an effective probe of the different angular correlations in the two initial angular states. With this aim, we fix $\rho_{E}^{1}(0)$ and $\rho_{E}^{2}(0)$ as the states corresponding to $\Delta \lambda_{1}=45 \mathrm{~nm}$ and $\Delta \lambda_{2}=10 \mathrm{~nm}$, respectively, that is a weakly and a strongly correlated angular state, while we consider different pairs of initial polarization states. To this end we set $\alpha=\pi / 4$ for both $\rho_{s}^{1}(0)$ and $\rho_{S}^{2}(0)$, and keep $\gamma_{1}$ fixed, while we vary $\gamma_{2}$ by inserting different precompensation crystals. Specifically, we exploit a $3 \mathrm{~mm}$ crystal to fully compensate the delay time $\Delta \tau$ [34], a $1 \mathrm{~mm}$ crystal to partially compensate it, and also consider the case without any precompensation crystal.

The experimental data, together with the theoretical prediction obtained by Eq. (15), are shown in Fig. 3(a). For high values of $\gamma_{2}$, the trace distance between polarization states actually satisfies Eq. (4) and thus witnesses the different initial conditions in the angular degrees of freedom. The information due to the differences in $\rho_{E}^{1}(0)$ and $\rho_{E}^{2}(0)$ flows to the polarization degrees of freedom because of the engineered interaction. Thus, one can access through simple visibility measurements on the open system some information which was initially outside it. On the other hand, the revival of the trace distance above its initial value decreases with the decreasing of $\gamma_{2}$, and for low enough values of $\gamma_{2}$ the trace distance remains below its initial value for the whole evolution. The loss of purity and entanglement due to a decrease of the parameter $\gamma$ in the initial polarization states can prevent the subsequent trace distance from being an effective probe of the different correlations in the angular states.

The relative weight of vertically and horizontally polarized photons generated by SPDC is determined by the parameter $\alpha$, which can be controlled by properly rotating a half-wave plate set in the pump beam. In Fig. 3(b) we report the experimental data and theoretical predictions of the trace-distance behavior for a given value of $\alpha_{1}$ as well as fixed $\gamma_{1}$ and $\gamma_{2}$, while considering different values of $\alpha_{2}$. One can see that, even if the growth of the trace distance above its initial value decreases with the decrease of $\alpha_{2}$, it is still visible also for a sensible imbalance between vertically and horizontally polarized photons. Indeed, for a fixed $\gamma<1$ the decrease of $\sin (2 \alpha)$ in the polarization states $\rho_{S}^{k}(0)$ corresponds to a decrease of the concurrence, but to an increase of the purity; see Eqs. (10) and (11). Contrary to what happens for a decrease of the parameter $\gamma_{2}$ [see Fig. 3(a)] the open system always 

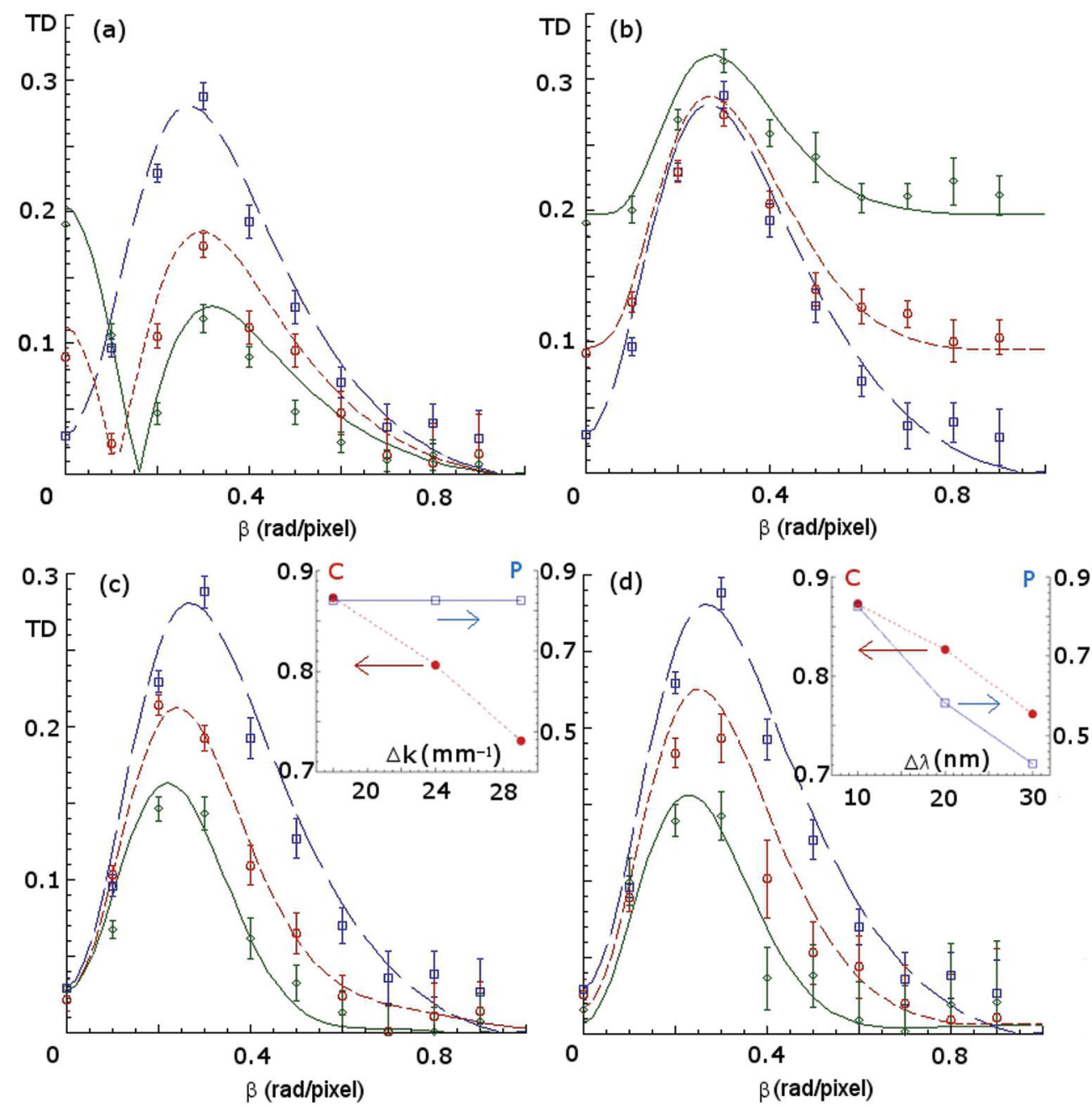

FIG. 3. (Color online) Trace distance $D(\beta)$ versus the evolution parameter $\beta$ for different pairs of initial system states and different environmental correlations [see Eq. (7)], showing how the trace-distance growth is sensitive to the environmental correlations. (a), (b) $\rho_{E}^{1}(0)$ and $\rho_{E}^{2}(0)$ are kept fixed $\left(\Delta k_{1}=\Delta k_{2}=18 \mathrm{~mm}^{-1}, \Delta \lambda_{1}=45\right.$, and $\left.\Delta \lambda_{2}=10 \mathrm{~nm}\right), \rho_{s}^{1}(0)$ is fixed with $\alpha_{1}=\pi / 4$ and $\gamma_{1}=0.91$ (mainly due to contributions to the phase in Eq. (1) which are not compensated to the first order), $\rho_{S}^{2}(0)$ corresponds to $\alpha_{2}=\pi / 4$ and $\gamma_{2}=0.96$ (blue long-dashed line), 0.73 (red short-dashed line), 0.52 (green solid line) in (a), while $\gamma_{2}=0.96$ and $\alpha_{2}=\pi / 4$ (blue long-dashed line), 0.675 (red short-dashed line), 0.575 (green solid line) in (b). (c), (d) $\rho_{S}^{1}(0)$ and $\rho_{S}^{2}(0)$ are fixed $\left(\alpha_{1}=\alpha_{2}=\pi / 4, \gamma_{1}=0.91\right.$, and $\left.\gamma_{2}=0.96\right), \rho_{E}^{1}(0)$ is fixed with $\Delta \lambda_{1}=45 \mathrm{~nm}$ and $\Delta k_{1}=18 \mathrm{~mm}^{-1}$, while $\rho_{E}^{2}(0)$ corresponds to $\Delta \lambda_{2}=10 \mathrm{~nm}$ and $\Delta k_{2}=18 \mathrm{~mm}^{-1}$ (blue long-dashed line), $24 \mathrm{~mm}^{-1}$ (red short-dashed line), $29 \mathrm{~mm}^{-1}$ (green solid line) in (c) and to $\Delta k_{2}=18 \mathrm{~mm}^{-1}$ and $\Delta \lambda_{2}=10 \mathrm{~nm}$ (blue long-dashed line), $20 \mathrm{~nm}$ (red short-dashed line), $30 \mathrm{~nm}$ (green solid line) in (d). The insets in (c) and (d) show the angular correlations (red dashed line) and the purity (blue solid line) of $\rho_{E}^{2}(0)$ as a function of $\Delta k_{2}$ [in (c)] or $\Delta \lambda_{2}$ [in (d)]. Experimental data are reported with their error bars, the lines represent the theoretical predictions.

recovers the information initially outside it from the very beginning of its evolution and the trace-distance maximum increases with the increase of the initial distinguishability between the two polarization states.

\section{B. Trace-distance dependence on the initial correlations in the angular degrees of freedom}

The analysis of the previous section shows that the optimal probe of the angular correlations is achieved by exploiting the highest amount of purity and entanglement of the polarization degrees of freedom available within our setting. Now, we study how this optimal probe is related to the changes in the angular correlations. Hence, we fix $\rho_{s}^{1}(0)$ and $\rho_{s}^{2}(0)$ and we investigate the trace-distance evolution $D\left(\rho_{s}^{1}(\beta), \rho_{s}^{2}(\beta)\right)$ for different pairs of initial angular states. In particular, we take as reference environmental state $\rho_{E}^{1}(0)$ the state with weak angular correlations, which is obtained by means of a collimated beam and a $45 \mathrm{~nm}$ spectrum. We compare the evolution of the subsequent polarization state $\rho_{s}^{1}(\beta)$ with the evolution of a state $\rho_{s}^{2}(\beta)$ evolved in the presence of strong initial angular correlations in $\rho_{E}^{2}(0)$. We repeat this procedure by changing the amount of correlation $C$ in $\rho_{E}^{2}(0)$ [see Eq. (7)], thus studying the connection between $C$ and the effectiveness of the quantum probe of the angular correlations quantified by the increase of the trace distance above its initial value.

In Fig. 3(c), one can see the experimental data and theoretical prediction concerning the different trace-distance 


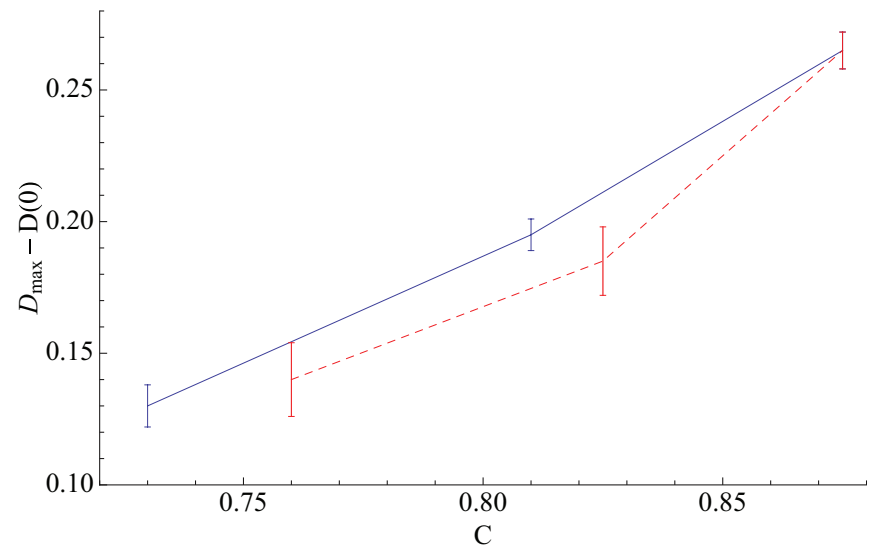

FIG. 4. (Color online) Experimental values of the maximum increase of the trace distance above its initial value as a function of the angular correlations $C$; see Eq. (7). The experimental data are referred to the behavior of the trace distance for different beam divergences [blue solid line, compare with Fig. 3(c)] or different widths of the frequency spectrum [red dashed line, compare with Fig. 3(d)].

evolutions $D\left(\rho_{s}^{1}(\beta), \rho_{s}^{2}(\beta)\right)$ which correspond to the different beam divergences exploited, together with a $10 \mathrm{~nm}$ spectrum, in the preparation of the environmental state $\rho_{E}^{2}(0)$. The divergence is enlarged by suitably setting a telescopic system of lenses, so that the full width at half maximum (FWHM) $\Delta k$ of $\left|\tilde{F}\left(\Delta k_{\perp}\right)\right|^{2}$ is increased, while the $220 \mu \mathrm{m}$ spot on the generating crystals is kept fixed [31]. The increase of the trace distance above its initial value grows with the angular correlations $C$ in $\rho_{E}^{2}(0)$. The behavior of $D\left(\rho_{S}^{1}(\beta), \rho_{S}^{2}(\beta)\right)$ indicates that, for the specific choice of $\rho_{S}^{1}(0)$ and $\rho_{S}^{2}(0)$, the trace distance is actually sensitive even to a small difference in the angular correlations of $\rho_{E}^{1}(0)$ and $\rho_{E}^{2}(0)$. The direct connection between the trace distance and the correlations in the environment is further shown in Fig. 4, where the difference between the maximum and the initial value of the trace distance is plotted as a function of the angular correlations. The experimental data indicate that the trace distance probes the different initial conditions in the environment, according to Eq. (4). In addition, for the model at hand, such a probe reflects the different amounts of correlation within the environment, which is indeed not $a$ priori entailed by Eq. (4).

As a further check of the connection between angular correlations and the increase of the trace distance above its initial value, we take into account environmental states $\rho_{E}^{2}(0)$ in which the angular correlations are modified by selecting different frequency spectra of the two-photon state. Besides affecting the angular correlations, this also influences the purity of $\rho_{E}^{2}(0)$. As may be inferred from Eqs. (5) and (6), a wider frequency spectrum implies a lower angular purity, as a consequence of the fact that the pure state generated by SPDC in Eq. (1) also involves the frequencies. The insets in Figs. 3(c) and 3(d), and Fig. 4 show that the growth of the trace distance above its initial value is not affected by the different purities of $\rho_{E}^{2}(0)$ in the two situations, but is determined by the amount of angular correlation $C$. In fact, analyzing Eqs. (14) and (15), one can see how the trace-distance evolution does not keep track of the purity of the angular states. The distinguishability between polarization states solely depends on the angular probability distribution $P\left(\theta_{s}, \theta_{i}\right)$, while it is independent of angular coherences, which fix the purity of the environmental state. From a physical point of view, this corresponds to the fact that the information which can be transferred from the environment to the system via the engineered interaction in Eq. (12) is stored in the angular correlations and not in the angular purity.

\section{CONCLUSION}

We have theoretically described and experimentally demonstrated a strategy to assess relevant information about a composite system by observing only a small and easily accessible part of it. By exploiting pairs of entangled photons generated by SPDC and engineering a proper interaction by means of a SLM, correlations within the angular degrees of freedom of the photons have been related to the trace-distance evolution between pairs of polarization states. After estimating the optimal probe, we have shown that the increase of the trace distance between system states above its initial value provides a signature of the amount of angular correlation in the environmental states.

\section{ACKNOWLEDGMENTS}

This work has been supported by the COST Action MP1006, the MIUR Project FIRB LiCHIS-RBFR10YQ3H, and the UniMI Project SIN.PHO.NANO. S.C. and M.G.A.P. would like to thank F. Ragusa for his support during the early stage of this work.
[1] C. J. Myatt, B. E. King, Q. A. Turchette, C. A. Sackett, D. Kielpinski, W. M. Itano, C. Monroe, and D. J. Wineland, Nature (London) 403, 269 (2000).

[2] B. Kraus and J. I. Cirac, Phys. Rev. Lett. 92, 013602 (2004).

[3] C. W. Chou, H. de Riedmatten, D. Felinto, S. V. Polyakov, S. J. van Enk, and H. J. Kimble, Nature (London) 438, 828 (2005).

[4] F. Verstraete, M. M. Wolf, and J. I. Cirac, Nat. Phys. 5, 633 (2009).

[5] J. T. Barreiro, M. Müller, P. Schindler, D. Nigg, T. Monz, M. Chwalla, M. Hennrich, C. F. Roos, P. Zoller, and R. Blatt, Nature (London) 470, 486 (2011).
[6] H. Krauter, C. A. Muschik, K. Jensen, W. Wasilewski, J. M. Petersen, J. I. Cirac, and E. S. Polzik, Phys. Rev. Lett. 107, 080503 (2011).

[7] G. Brida, I. P. Degiovanni, A. Florio, M. Genovese, P. Giorda, A. Meda, M. G. A. Paris, and A. Shurupov, Phys. Rev. Lett. 104, 100501 (2010).

[8] R. Blandino, M. G. Genoni, J. Etesse, M. Barbieri, M. G. A. Paris, P. Grangier, and R. Tualle-Brouri, Phys. Rev. Lett 109, 180402 (2012).

[9] C. K. Hong and L. Mandel, Phys. Rev. A 31, 2409 (1985). 
[10] A. Joobeur, B. E. A. Saleh, and M. C. Teich, Phys. Rev. A 50, 3349 (1994).

[11] P. G. Kwiat, E. Waks, A. G. White, I. Appelbaum, and P. H. Eberhard, Phys. Rev. A 60, R773 (1999).

[12] C. C. Gerry and P. L. Knight, Introductory Quantum Optics (Cambridge University Press, Cambridge, 2005).

[13] H.-P. Breuer, E.-M. Laine, and J. Piilo, Phys. Rev. Lett. 103, 210401 (2009); E.-M. Laine, J. Piilo, and H.-P. Breuer, Phys. Rev. A 81, 062115 (2010).

[14] T. J. G. Apollaro, C. Di Franco, F. Plastina, and M. Paternostro, Phys. Rev. A 83, 032103 (2011).

[15] B. H. Liu, L. Li, Y.-F. Huang, C.-F. Li, G.-C. Guo, E.-M. Laine, H.-P. Breuer, and J. Piilo, Nat. Phys. 7, 931 (2011).

[16] B. Vacchini, A. Smirne, E.-M. Laine, J. Piilo, and H.-P. Breuer, New. J. Phys. 13, 093004 (2011).

[17] H.-P. Breuer, J. Phys. B 45, 15 (2012).

[18] A. Smirne, L. Mazzola, M. Paternostro, and B. Vacchini, Phys. Rev. A 87, 052129 (2013).

[19] E.-M. Laine, J. Piilo, and H.-P. Breuer, Europhys. Lett. 92, 60010 (2010).

[20] A. Smirne, D. Brivio, S. Cialdi, B. Vacchini, and M. G. A. Paris, Phys. Rev. A 84, 032112 (2011).

[21] J. Dajka, J. Łuczka, and P. Hänggi, Phys. Rev. A 84, 032120 (2011).

[22] M. Gessner and H.-P. Breuer, Phys. Rev. Lett. 107, 180402 (2011).

[23] E.-M. Laine, H.-P. Breuer, J. Piilo, C.-F. Li, and G.-C. Guo, Phys. Rev. Lett. 108, 210402 (2012).

[24] B. H. Liu, D.-Y. Cao, Y.-F. Huang, C.-F. Li, G.-C. Guo, E.-M. Laine, H.-P. Breuer, and J. Piilo, Sci. Rep. 3, 1781 (2013).
[25] E.-M. Laine, H.-P. Breuer, and J. Piilo, arXiv:1210.8266.

[26] P. Haikka, S. McEndoo, G. De Chiara, G. M. Palma, and S. Maniscalco, Phys. Rev. A 84, 031602(R) (2011).

[27] P. Haikka, J. Goold, S. McEndoo, F. Plastina, and S. Maniscalco, Phys. Rev. A 85, 060101(R) (2012).

[28] P. Haikka, S. McEndoo, and S. Maniscalco, Phys. Rev. A 87, 012127 (2013).

[29] R. Rangarajan, M. Goggin, and P. Kwiat, Opt. Express 17, 18920 (2009).

[30] S. Cialdi, D. Brivio, and M. G. A. Paris, Appl. Phys. Lett. 97, 041108 (2010)

[31] S. Cialdi, D. Brivio, A. Tabacchini, A. M. Kadhim, and M. G. A. Paris, Opt. Lett. 37, 3951 (2012).

[32] S. Cialdi, D. Brivio, and M. G. A. Paris, Phys. Rev. A 81, 042322 (2010).

[33] S. Cialdi, D. Brivio, E. Tesio, and M. G. A. Paris, Phys. Rev. A 83, 042308 (2011).

[34] S. Cialdi, F. Castelli, I. Boscolo, and M. G. A. Paris, Appl. Opt. 47, 1832 (2008).

[35] C. A. Fuchs and J. van de Graaf, IEEE Trans. Inf. Theory 45, 1216 (1999).

[36] H.-P. Breuer and F. Petruccione, The Theory of Open Quantum Systems (Oxford University Press, Oxford, 2002).

[37] A. Smirne, H. P. Breuer, J. Piilo, and B. Vacchini, Phys. Rev. A 82, 062114 (2010).

[38] S. Hill and W. K. Wootters, Phys. Rev. Lett. 78, 5022 (1997).

[39] S. Cialdi, F. Castelli, and M. G. A. Paris, J. Mod. Opt. 56, 215 (2009). 